\title{
Standing Wave Solutions for the Discrete Coupled Nonlinear Schrödinger Equations with Unbounded Potentials
}

\author{
Meihua Huang ${ }^{1,2}$ and Zhan Zhou ${ }^{1,2}$ \\ ${ }^{1}$ School of Mathematics and Information Science, Guangzhou University, Guangzhou, Guangdong 510006, China \\ ${ }^{2}$ Key Laboratory of Mathematics and Interdisciplinary Sciences of Guangdong Higher Education Institutes, \\ Guangzhou University, Guangzhou, Guangdong 510006, China
}

Correspondence should be addressed to Zhan Zhou; zzhou0321@hotmail.com

Received 18 January 2013; Accepted 25 February 2013

Academic Editor: Chuangxia Huang

Copyright (C) 2013 M. Huang and Z. Zhou. This is an open access article distributed under the Creative Commons Attribution License, which permits unrestricted use, distribution, and reproduction in any medium, provided the original work is properly cited.

We demonstrate the existence of standing wave solutions of the discrete coupled nonlinear Schrödinger equations with unbounded potentials by using the Nehari manifold approach and the compact embedding theorem. Sufficient conditions are established to show that the standing wave solutions have both of the components not identically zero.

\section{Introduction}

Consider the coupled discrete Schrödinger system

$$
\begin{aligned}
& i \frac{d u_{n}}{d t}=-(\mathscr{A} u)_{n}+b_{1 n} u_{n}-a_{1}\left|u_{n}\right|^{2} u_{n}-a_{3}\left|v_{n}\right|^{2} u_{n} \\
& i \frac{d v_{n}}{d t}=-(\mathscr{A} v)_{n}+b_{2 n} v_{n}-a_{2}\left|v_{n}\right|^{2} v_{n}-a_{3}\left|u_{n}\right|^{2} v_{n}
\end{aligned}
$$

where $a_{i}>0,\left\{b_{j n}\right\}$ are real valued sequences, $i=$ $1,2,3$, and $j=1,2$. $\mathscr{A}$ is the discrete Laplacian operator defined as $(\mathscr{A} u)_{n}=u_{n+1}+u_{n-1}-2 u_{n}$.

The system (1) could be viewed as the discretization of the two-component system of time-dependent nonlinear GrossPitaevskii system (see [1] for detail)

$$
\begin{aligned}
i \hbar \partial_{t} u & =-\frac{\hbar^{2}}{2 m} \Delta u+b_{1}(x) u-a_{1}|u|^{2} u-a_{3}|v|^{2} u, \\
i \hbar \partial_{t} v & =-\frac{\hbar^{2}}{2 m} \Delta v+b_{2}(x) v-a_{2}|v|^{2} v-a_{3}|u|^{2} v .
\end{aligned}
$$

In this paper, we will study the standing wave solutions of (1), that is, solutions of the form

$$
u_{n}=\exp \left(-i \omega_{1} t\right) \phi_{n}, \quad v_{n}=\exp \left(-i \omega_{2} t\right) \psi_{n}, \quad n \in \mathbb{Z},
$$

where the amplitude $\phi_{n}$ and $\psi_{n}$ are supposed to be real. Inserting the ansatz of the standing wave solutions (3) into (1), we obtain the following equivalent algebraic equations:

$$
\begin{aligned}
& -(\mathscr{A} \phi)_{n}-\omega_{1} \phi_{n}+b_{1 n} \phi_{n}-a_{1}\left|\phi_{n}\right|^{2} \phi_{n}-a_{3}\left|\psi_{n}\right|^{2} \phi_{n}=0, \\
& -(\mathscr{A} \psi)_{n}-\omega_{2} \psi_{n}+b_{2 n} \psi_{n}-a_{2}\left|\psi_{n}\right|^{2} \psi_{n}-a_{3}\left|\phi_{n}\right|^{2} \psi_{n}=0 .
\end{aligned}
$$

Since Bose-Einstein condensation for a mixture of different interaction atomic species with the same mass was realized in 1997 (see [2]), this stimulated various analytical and numerical results on the standing wave solutions of the system (2). The discrete nonlinear Schrödinger equations (DNLS) have a crucial role in the modeling of a great variety of phenomena, ranging from solid-state and condensedmatter physics to biology. During the last years, there has been a growing interest in approaches to the existence problem for standing waves. We refer to the continuation methods in $[3,4]$, which have been proved powerful for both theoretical considerations and numerical computations (see [5]), to [6], which exploits spatial dynamics and centre manifold reduction, and to the variational methods in [7-11], which rely on critical point techniques (linking theorems and Nehari manifold).

We noticed that most works on the existence of standing waves solutions are for single discrete nonlinear 
Schrödinger equation, and less is known for discrete nonlinear Schrödinger system. In the recent paper [12], the authors considered the standing wave solutions of the following system:

$$
\begin{aligned}
& i \frac{d u_{n}}{d t}=-(\mathscr{A} u)_{n}+b_{1 n} u_{n}-c_{1 n} v_{n}-a_{1}\left|u_{n}\right|^{2} u_{n}-a_{3}\left|v_{n}\right|^{2} u_{n} \\
& i \frac{d v_{n}}{d t}=-(\mathscr{A} v)_{n}+b_{2 n} v_{n}-c_{2 n} u_{n}-a_{2}\left|v_{n}\right|^{2} v_{n}-a_{3}\left|u_{n}\right|^{2} v_{n}
\end{aligned}
$$

which is more general than the system (1). However, they make a mistake to obtain the equivalent algebraic equations because $\omega_{1}$ may be different from $\omega_{2}$. Hence, there are two ways to correct this mistake. The first method is to study the special standing wave solutions (3) of the system (5) with $\omega_{1}=$ $\omega_{2}$. The second method is to study the standing wave solutions (3) of the system (5) with $c_{1 n} \equiv 0$ and $c_{2 n} \equiv 0, n \in \mathbb{Z}$. In this paper, we consider the second method. By the way, the proof of the main results in [12] is also not fully corrected.

The paper is organized as follows. In Section 2, we introduce some preliminaries and a discrete version of compact embedding theorem. Some key lemmas on the Nehari manifold are proved in Section 3. In Section 4, the main results are stated and proved.

\section{Preliminaries}

In this section we describe the functional setting needed for the treatment of the infinite nonlinear system (4). We first introduce a compact embedding theorem.

Consider the real sequence spaces

$$
\begin{gathered}
l^{p}=\left\{\phi=\left\{\phi_{n}\right\}_{n \in \mathbb{Z}}:\|\phi\|_{l^{p}}=\left(\sum_{n \in \mathbb{Z}}\left|\phi_{n}\right|^{p}\right)^{1 / p}<\infty,\right. \\
\left.\phi_{n} \in \mathbb{R}, \forall n \in \mathbb{Z}\right\} .
\end{gathered}
$$

Between $l^{p}$ spaces the following elementary embedding relation holds:

$$
l^{q} \subset l^{p}, \quad\|\phi\|_{l^{p}} \leq\|\phi\|_{l^{q}}, \quad 1 \leq q \leq p \leq \infty .
$$

For the case $p=2$, we need the usual Hilbert space of $l^{2}$, endowed with the real scalar product

$$
(\phi, \psi)=\sum_{n \in \mathbb{Z}} \phi_{n} \psi_{n}, \quad \phi, \psi \in l^{2} .
$$

Let us point out that the spectrum of $-\mathscr{A}$ in $l^{2}$ coincides with the interval $[0,4]$. Obviously, we have

$$
0 \leq(-\mathscr{A} \phi, \phi) \leq 4\|\phi\|_{l^{2}}^{2}, \quad \forall \phi \in l^{2} .
$$

Assume that the potential $V_{i}=\left\{b_{i n}\right\}_{n \in \mathbb{Z}}, i=1,2$, satisfies

$$
\lim _{|n| \rightarrow \infty} b_{i n}=\infty, \quad i=1,2 .
$$

Without loss of generality we assume that $V_{i} \geq 1, i=1,2$; that is $b_{\text {in }} \geq 1$ for $n \in \mathbb{Z}, i=1,2$. Let

$$
L_{i}=-\mathscr{A}+V_{i}, \quad i=1,2,
$$

which are self-adjoint operators defined on $l^{2}$, and

$$
\begin{array}{r}
E_{i}=\left\{\phi \in l^{2}: L_{i}^{1 / 2} \phi \in l^{2}\right\}, \quad\|\phi\|_{E_{i}}=\left\|L_{i}^{1 / 2} \phi\right\|_{l^{2}}, \\
i=1,2 .
\end{array}
$$

The following lemma can be found in [9].

Lemma 1. If $V_{i}, i=1,2$, satisfy the condition (10), then for any $2 \leq p \leq \infty, E_{1}$ and $E_{2}$ are compactly embedded into $l^{p}$ and denote the best embedding constant $\alpha_{p}=\max _{\|\phi\|_{l}=1} 1 /\|\phi\|_{E_{1}}$ and $\beta_{p}=\max _{\|\phi\|_{1}=1} 1 /\|\phi\|_{E_{2}}$, respectively. Furthermore, the spectra $\sigma\left(L_{1}\right)$ and $\sigma\left(L_{2}\right)$ are discrete, respectively.

By (11), (4) becomes

$$
\begin{aligned}
& L_{1} \phi_{n}-\omega_{1} \phi_{n}-a_{1}\left|\phi_{n}\right|^{2} \phi_{n}-a_{3}\left|\psi_{n}\right|^{2} \phi_{n}=0, \\
& L_{2} \psi_{n}-\omega_{2} \psi_{n}-a_{2}\left|\psi_{n}\right|^{2} \psi_{n}-a_{3}\left|\phi_{n}\right|^{2} \psi_{n}=0 .
\end{aligned}
$$

Now we can define the action functional

$$
\begin{aligned}
J(\phi, \psi)= & \frac{1}{2}\left(\left(L_{1}-\omega_{1}\right) \phi, \phi\right)+\frac{1}{2}\left(\left(L_{2}-\omega_{2}\right) \psi, \psi\right) \\
& -\frac{1}{4} \sum_{n \in \mathbb{Z}}\left(a_{1} \phi_{n}^{4}+a_{2} \psi_{n}^{4}+2 a_{3} \phi_{n}^{2} \psi_{n}^{2}\right) .
\end{aligned}
$$

By Lemma 1, it follows that the action functional $J(\phi, \psi) \in$ $C^{1}\left(E_{1} \times E_{2}, \mathbb{R}\right)$ and (13) corresponds to $J^{\prime}(\phi, \psi)=0$. So we define

$$
\begin{aligned}
I(\phi, \psi)= & \left(J^{\prime}(\phi, \psi),(\phi, \psi)\right) \\
= & \left(\left(L_{1}-\omega_{1}\right) \phi, \phi\right)+\left(\left(L_{2}-\omega_{2}\right) \psi, \psi\right) \\
& -\sum_{n \in \mathbb{Z}}\left(a_{1} \phi_{n}^{4}+a_{2} \psi_{n}^{4}+2 a_{3} \phi_{n}^{2} \psi_{n}^{2}\right),
\end{aligned}
$$

and the Nehari manifold

$$
N=\left\{(\phi, \psi) \in E_{1} \times E_{2}: I(\phi, \psi)=0,(\phi, \psi) \neq 0\right\} .
$$

\section{Some Lemmas on the Nehari Manifold}

Let

$$
\lambda_{i}=\inf \left\{\sigma\left(L_{i}\right)\right\}, \quad i=1,2
$$

To prove the main results, we need some lemmas on the Nehari manifold.

Lemma 2. Assume that $\omega_{1}<\lambda_{1}, \omega_{2}<\lambda_{2}$, and (10) holds. Then the Nehari manifold $N$ is nonempty in $E_{1} \times E_{2}$. Furthermore, for $(\phi, \psi) \in N, J(t \phi, t \psi)$ attains a unique maximum point at $t=1$. 
Proof. First we show that $N \neq \emptyset$.

From (15) and (16), we rewrite

$$
\begin{aligned}
J(\phi, \psi)= & \frac{1}{2}\left(\|\phi\|_{E_{1}}^{2}-\omega_{1}\|\phi\|_{l^{2}}^{2}\right) \\
& +\frac{1}{2}\left(\|\psi\|_{E_{2}}^{2}-\omega_{2}\|\psi\|_{l^{2}}^{2}\right) \\
& -\frac{1}{4} \sum_{n \in \mathbb{Z}}\left(a_{1} \phi_{n}^{4}+a_{2} \psi_{n}^{4}+2 a_{3} \phi_{n}^{2} \psi_{n}^{2}\right), \\
I(\phi, \psi)= & \|\phi\|_{E_{1}}^{2}-\omega_{1}\|\phi\|_{l^{2}}^{2}+\|\psi\|_{E_{2}}^{2}-\omega_{2}\|\psi\|_{l^{2}}^{2} \\
& -\sum_{n \in \mathbb{Z}}\left(a_{1} \phi_{n}^{4}+a_{2} \psi_{n}^{4}+2 a_{3} \phi_{n}^{2} \psi_{n}^{2}\right) .
\end{aligned}
$$

Let $(\phi, \psi) \in\left(E_{1}-\{0\}\right) \times\left(E_{2}-\{0\}\right)$; then by (19)

$$
\begin{aligned}
I(t \phi, t \psi)= & t^{2}\left(\|\phi\|_{E_{1}}^{2}-\omega_{1}\|\phi\|_{l^{2}}^{2}\right) \\
& +t^{2}\left(\|\psi\|_{E_{2}}^{2}-\omega_{2}\|\psi\|_{l^{2}}^{2}\right) \\
& -t^{4} \sum_{n \in \mathbb{Z}}\left(a_{1} \phi_{n}^{4}+a_{2} \psi_{n}^{4}+2 a_{3} \phi_{n}^{2} \psi_{n}^{2}\right) \\
= & t^{2}\left(\|\phi\|_{E_{1}}^{2}-\omega_{1}\|\phi\|_{l^{2}}^{2}+\|\psi\|_{E_{2}}^{2}-\omega_{2}\|\psi\|_{l^{2}}^{2}\right. \\
& \left.-t^{2} \sum_{n \in \mathbb{Z}}\left(a_{1} \phi_{n}^{4}+a_{2} \psi_{n}^{4}+2 a_{3} \phi_{n}^{2} \psi_{n}^{2}\right)\right) .
\end{aligned}
$$

Notice that $\|\phi\|_{E_{1}}^{2}-\omega_{1}\|\phi\|_{l^{2}}^{2} \geq\left(\lambda_{1}-\omega_{1}\right)\|\phi\|_{l^{2}}^{2}>0$ and $\|\psi\|_{E_{2}}^{2}-\omega_{2}\|\psi\|_{l^{2}}^{2} \geq\left(\lambda_{2}-\omega_{2}\right)\|\psi\|_{l^{2}}^{2}>0$; by (20), we see that $I(t \phi, t \psi)>0$ for $t>0$ small enough and $I(t \phi, t \psi)<0$ for $t>0$ large enough. As a consequence, there exists $t_{0}>0$ such that $I\left(t_{0} \phi, t_{0} \psi\right)=0$; that is, $\left(t_{0} \phi, t_{0} \psi\right) \in N$.

Let $F(t)=J(t \phi, t \psi),(\phi, \psi) \in N$. Computing the derivative of $F$, we have

$$
F^{\prime}(t)=t\left(1-t^{2}\right) \sum_{n \in \mathbb{Z}}\left(a_{1} \phi_{n}^{4}+a_{2} \psi_{n}^{4}+2 a_{3} \phi_{n}^{2} \psi_{n}^{2}\right)
$$

This shows that $t=1$ is a unique maximum point. The proof is completed.

Lemma 3. Assume that $\omega_{1}<\lambda_{1}, \omega_{2}<\lambda_{2}$, and (10) holds. Then there exists $\eta>0$ such that $J(\phi, \psi) \geq \eta$, for all $(\phi, \psi) \in N$.

Proof. Since $\lambda_{1}$ is the smallest eigenvalue of $E_{1}$ and $\lambda_{2}$ is the smallest eigenvalue of $E_{2}$, from the definition of the constant $\alpha_{p}$ and $\beta_{p}$, we get $\lambda_{1}=1 / \alpha_{2}^{2}$ and $\lambda_{2}=1 / \beta_{2}^{2}$. For any $(\phi, \psi) \epsilon$ $N$, we have

$$
\begin{aligned}
\|\phi\|_{E_{1}}^{2} & -\omega_{1}\|\phi\|_{l^{2}}^{2}+\|\psi\|_{E_{2}}^{2}-\omega_{2}\|\psi\|_{l^{2}}^{2} \\
& =\sum_{n \in \mathbb{Z}}\left(a_{1} \phi_{n}^{4}+a_{2} \psi_{n}^{4}+2 a_{3} \phi_{n}^{2} \psi_{n}^{2}\right) \\
& \leq a^{*}\left(\|\phi\|_{l^{4}}^{2}+\|\psi\|_{l^{4}}^{2}\right)^{2} \\
& \leq a^{*}\left(\alpha_{4}^{2}\|\phi\|_{E_{1}}^{2}+\beta_{4}^{2}\|\psi\|_{E_{2}}^{2}\right)^{2} \\
& \leq a^{*} \gamma_{2}^{2}\left(\|\phi\|_{E_{1}}^{2}+\|\psi\|_{E_{2}}^{2}\right)^{2},
\end{aligned}
$$

where $a^{*}=\max \left\{a_{1}, a_{2}, a_{3}\right\}$ and $\gamma_{2}=\max \left\{\alpha_{4}^{2}, \beta_{4}^{2}\right\}$.

Let

$$
\gamma_{1}=\min \left\{1,1-\frac{\omega_{1}}{\lambda_{1}}, 1-\frac{\omega_{2}}{\lambda_{2}}\right\} .
$$

By (22), it is easy to see that

$$
\gamma_{1}\left(\|\phi\|_{E_{1}}^{2}+\|\psi\|_{E_{2}}^{2}\right) \leq a^{*} \gamma_{2}^{2}\left(\|\phi\|_{E_{1}}^{2}+\|\psi\|_{E_{2}}^{2}\right)^{2}
$$

and this implies that

$$
\|\phi\|_{E_{1}}^{2}+\|\psi\|_{E_{2}}^{2} \geq \frac{\gamma_{1}}{a^{*} \gamma_{2}^{2}} .
$$

Moreover, we have

$$
\begin{aligned}
J(\phi, \psi) & =J(\phi, \psi)-\frac{1}{4} I(\phi, \psi) \\
& =\frac{1}{4}\left(\|\phi\|_{E_{1}}^{2}-\omega_{1}\|\phi\|_{l^{2}}^{2}+\|\psi\|_{E_{2}}^{2}-\omega_{2}\|\psi\|_{l^{2}}^{2}\right) \\
& \geq \frac{\gamma_{1}}{4}\left(\|\phi\|_{E_{1}}^{2}+\|\psi\|_{E_{2}}^{2}\right) \geq \frac{\gamma_{1}^{2}}{4 a^{*} \gamma_{2}^{2}} .
\end{aligned}
$$

Let $\eta=\gamma_{1}^{2} /\left(4 a^{*} \gamma_{2}^{2}\right)$; then we get $J(\phi, \psi) \geq \eta$, for all $(\phi, \psi) \in N$. The proof is completed.

\section{Main Results}

Now we state our main results in this paper as follows.

Theorem 4. Assume that $\omega_{1}<\lambda_{1}, \omega_{2}<\lambda_{2}$, and (10) holds. Then system (13) has a nontrivial solution in $E_{1} \times E_{2}$; that is, system (1) has a nontrivial standing wave solution.

In order to prove Theorem 4, we consider the following constrained minimization problem:

$$
d \equiv \inf _{(\phi, \psi) \in N} J(\phi, \psi)
$$

From the standard variational method, the proof of Theorem 4 is changed into finding a solution to the minimization problem (27). Now we are ready to prove Theorem 4. 
Proof. Let $d$ be given by (27). By Lemma 2, $N$ is nonempty and there exists a sequence $\left\{\left(\phi^{(k)}, \psi^{(k)}\right)\right\} \subset N$ such that

$$
d=\lim _{k \rightarrow \infty} J\left(\phi^{(k)}, \psi^{(k)}\right) .
$$

By Lemma 3, $d>0$ and $d \leq D=\max _{k}\left\{J\left(\phi^{(k)}, \psi^{(k)}\right)\right\}<$ $\infty$. By virtue of (26), we have

$$
\left\|\phi^{(k)}\right\|_{E_{1}}^{2}+\left\|\psi^{(k)}\right\|_{E_{2}}^{2} \leq \frac{4}{\gamma_{1}} J\left(\phi^{(k)}, \psi^{(k)}\right) \leq \frac{4 D}{\gamma_{1}}<\infty .
$$

Thus, sequences $\left\{\phi^{(k)}\right\}$ and $\left\{\psi^{(k)}\right\}$ are bounded in Hilbert spaces $E_{1}$ and $E_{2}$, respectively. Therefore, there exist subsequences of $\left\{\phi^{(k)}\right\}$ and $\left\{\psi^{(k)}\right\}$ (denoted by itself) that weakly converge to some $\phi^{*} \in E_{1}$ and $\psi^{*} \in E_{2}$, respectively. By Lemma 1 , we get, for any $2 \leq p \leq \infty$,

$$
\lim _{k \rightarrow \infty} \phi^{(k)}=\phi^{*}, \quad \lim _{k \rightarrow \infty} \psi^{(k)}=\psi^{*}, \quad \text { in } l^{p}
$$

By virtue of (15) and (16), we have

$$
\begin{aligned}
J\left(\phi^{(k)},\right. & \left.\psi^{(k)}\right) \\
= & \frac{1}{2}\left(\left\|\phi^{(k)}\right\|_{E_{1}}^{2}-\omega_{1}\left\|\phi^{(k)}\right\|_{l^{2}}^{2}\right)+\frac{1}{2}\left(\left\|\psi^{(k)}\right\|_{E_{2}}^{2}-\omega_{2}\left\|\psi^{(k)}\right\|_{l^{2}}^{2}\right) \\
& -\frac{1}{4} \sum_{n \in \mathbb{Z}}\left(a_{1}\left(\phi_{n}^{(k)}\right)^{4}+a_{2}\left(\psi_{n}^{(k)}\right)^{4}+2 a_{3}\left(\phi_{n}^{(k)}\right)^{2}\left(\psi_{n}^{(k)}\right)^{2}\right) \\
= & \frac{1}{4} \sum_{n \in \mathbb{Z}}\left(a_{1}\left(\phi_{n}^{(k)}\right)^{4}+a_{2}\left(\psi_{n}^{(k)}\right)^{4}+2 a_{3}\left(\phi_{n}^{(k)}\right)^{2}\left(\psi_{n}^{(k)}\right)^{2}\right) .
\end{aligned}
$$

First, we claim that

$$
\begin{gathered}
\lim _{k \rightarrow \infty} \sum_{n \in \mathbb{Z}}\left(a_{1}\left(\phi_{n}^{(k)}\right)^{4}+a_{2}\left(\psi_{n}^{(k)}\right)^{4}+2 a_{3}\left(\phi_{n}^{(k)}\right)^{2}\left(\psi_{n}^{(k)}\right)^{2}\right) \\
=\sum_{n \in \mathbb{Z}}\left(a_{1}\left(\phi_{n}^{*}\right)^{4}+a_{2}\left(\psi_{n}^{*}\right)^{4}+2 a_{3}\left(\phi_{n}^{*}\right)^{2}\left(\psi_{n}^{*}\right)^{2}\right) .
\end{gathered}
$$

According to (30), it suffices to show that

$$
\lim _{k \rightarrow \infty} \sum_{n \in \mathbb{Z}}\left(\phi_{n}^{(k)}\right)^{2}\left(\psi_{n}^{(k)}\right)^{2}=\sum_{n \in \mathbb{Z}}\left(\phi_{n}^{*}\right)^{2}\left(\psi_{n}^{*}\right)^{2} .
$$

In fact,

$$
\begin{aligned}
\left|\sum_{n \in \mathbb{Z}}\left(\phi_{n}^{(k)}\right)^{2}\left(\psi_{n}^{(k)}\right)^{2}-\sum_{n \in \mathbb{Z}}\left(\phi_{n}^{*}\right)^{2}\left(\psi_{n}^{*}\right)^{2}\right| \\
\leq \sum_{n \in \mathbb{Z}}\left|\phi_{n}^{(k)}-\phi_{n}^{*}\right|\left|\phi_{n}^{(k)}+\phi_{n}^{*}\right|\left(\psi_{n}^{(k)}\right)^{2} \\
+\sum_{n \in \mathbb{Z}}\left|\psi_{n}^{(k)}-\psi_{n}^{*}\right|\left|\psi_{n}^{(k)}+\psi_{n}^{*}\right|\left(\phi_{n}^{*}\right)^{2} .
\end{aligned}
$$

Thus Hölder inequality and (30) imply the (33) holds.
Next, we show that $\left(\phi^{*}, \psi^{*}\right) \in N$ and $J\left(\phi^{*}, \psi^{*}\right)=d$. Since $E_{1}$ and $E_{2}$ are Hilbert spaces, by (32) we have

$$
\begin{aligned}
\left\|\phi^{*}\right\|_{E_{1}}^{2}+\left\|\psi^{*}\right\|_{E_{2}}^{2} & \| \text { weak }-\lim _{k \rightarrow \infty} \phi^{(k)}\left\|_{E_{1}}^{2}+\right\| \text { weak }-\lim _{k \rightarrow \infty} \psi^{(k)} \|_{E_{2}}^{2} \\
\leq & \liminf _{k \rightarrow \infty}\left\|\phi^{(k)}\right\|_{E_{1}}^{2}+\liminf _{k \rightarrow \infty}\left\|\psi^{(k)}\right\|_{E_{2}}^{2} \\
\leq & \liminf _{k \rightarrow \infty}\left(\left\|\phi^{(k)}\right\|_{E_{1}}^{2}+\left\|\psi^{(k)}\right\|_{E_{2}}^{2}\right) \\
= & \liminf _{k \rightarrow \infty}\left(\sum _ { n \in \mathbb { Z } } \left(a_{1}\left(\phi_{n}^{(k)}\right)^{4}+a_{2}\left(\psi_{n}^{(k)}\right)^{4}\right.\right. \\
& \left.+2 a_{3}\left(\phi_{n}^{(k)}\right)^{2}\left(\psi_{n}^{(k)}\right)^{2}\right) \\
& \left.+\omega_{1}\left\|\phi^{(k)}\right\|_{l_{2}}^{2}+\omega_{2}\left\|\psi^{(k)}\right\|_{l_{2}}^{2}\right) \\
& +\sum_{n \in \mathbb{Z}}\left(a_{1}\left(\phi_{n}^{*}\right)^{4}+a_{2}\left(\psi_{n}^{*}\right)^{4}+2 a_{3}\left(\phi_{n}^{*}\right)^{2}\left(\psi_{n}^{*}\right)^{2}\right) \\
& +\omega_{1}\left\|\phi^{*}\right\|_{l_{2}}^{2}+\omega_{2}\left\|\psi^{*}\right\|_{l_{2}}^{2},
\end{aligned}
$$

which implies $I\left(\phi^{*}, \psi^{*}\right)=\left\|\phi^{*}\right\|_{E_{1}}^{2}-\omega_{1}\left\|\phi^{*}\right\|_{l_{2}}^{2}+\left\|\psi^{*}\right\|_{E_{2}}^{2}-$ $\omega_{2}\left\|\psi^{*}\right\|_{l_{2}}^{2}-\sum_{n \in \mathbb{Z}}\left(a_{1}\left(\phi_{n}^{*}\right)^{4}+a_{2}\left(\psi_{n}^{*}\right)^{4}+2 a_{3}\left(\phi_{n}^{*}\right)^{2}\left(\psi_{n}^{*}\right)^{2}\right) \leq 0$. Through a similar argument to the proof of Lemma 2, we know that $I\left(t \phi^{*}, t \psi^{*}\right)$ is positive as $t$ is small enough. Therefore there exists $t^{*} \in(0,1]$ such that $I\left(t^{*} \phi^{*}, t^{*} \psi^{*}\right)=0$ which implies $\left(t^{*} \phi^{*}, t^{*} \psi^{*}\right) \in N$. Thus we have $J\left(t^{*} \phi^{*}, t^{*} \psi^{*}\right)=$ $(1 / 4) W\left(t^{*}\right)$ and by (32), $W(1)=4 d$, where

$$
W(t)=t^{4} \sum_{n \in \mathbb{Z}}\left(a_{1}\left(\phi_{n}^{*}\right)^{4}+a_{2}\left(\psi_{n}^{*}\right)^{4}+2 a_{3}\left(\phi_{n}^{*}\right)^{2}\left(\psi_{n}^{*}\right)^{2}\right)
$$

Clearly, $W(t)$ is strictly increasing on $0<t<\infty$. Therefore by $(27)$,

$$
d \leq J\left(t^{*} \phi^{*}, t^{*} \psi^{*}\right)=\frac{1}{4} W\left(t^{*}\right) \leq \frac{1}{4} W(1)=d .
$$

This implies that $t^{*}=1$ and $J\left(\phi^{*}, \psi^{*}\right)=d$.

Finally, we will prove $\left(\phi^{*}, \psi^{*}\right)$ is a nontrivial solution to system (13).

Since $\left(\phi^{*}, \psi^{*}\right)$ is an energy minimizer on Nehari manifold $N$, there exists a Lagrange multiplier $\Lambda$ such that

$$
\left(J^{\prime}\left(\phi^{*}, \psi^{*}\right)+\Lambda I^{\prime}\left(\phi^{*}, \psi^{*}\right),(\phi, \psi)\right)=0
$$

for any $(\phi, \psi) \in E_{1} \times E_{2}$. Let $(\phi, \psi)=\left(\phi^{*}, \psi^{*}\right)$ in (38). $\left(J^{\prime}\left(\phi^{*}, \psi^{*}\right),\left(\phi^{*}, \psi^{*}\right)\right)=I\left(\phi^{*}, \psi^{*}\right)=0$ implies that

$$
\Lambda\left(I^{\prime}\left(\phi^{*}, \psi^{*}\right),\left(\phi^{*}, \psi^{*}\right)\right)=0,
$$


but

$$
\begin{aligned}
\left(I^{\prime}\left(\phi^{*}, \psi^{*}\right),\left(\phi^{*}, \psi^{*}\right)\right) & \\
= & 2\left(\left(L_{1}-\omega_{1}\right) \phi^{*}, \phi^{*}\right)+2\left(\left(L_{2}-\omega_{2}\right) \psi^{*}, \psi^{*}\right) \\
& -4 \sum_{n \in \mathbb{Z}}\left(a_{1}\left(\phi_{n}^{*}\right)^{4}+a_{2}\left(\psi_{n}^{*}\right)^{4}+2 a_{3}\left(\phi_{n}^{*}\right)^{2}\left(\psi_{n}^{*}\right)^{2}\right) \\
= & -2 \sum_{n \in \mathbb{Z}}\left(a_{1}\left(\phi_{n}^{*}\right)^{4}+a_{2}\left(\psi_{n}^{*}\right)^{4}+2 a_{3}\left(\phi_{n}^{*}\right)^{2}\left(\psi_{n}^{*}\right)^{2}\right)<0 .
\end{aligned}
$$

Thus, $\Lambda=0$ and

$$
\left(J^{\prime}\left(\phi^{*}, \psi^{*}\right),(\phi, \psi)\right)=0,
$$

for any $(\phi, \psi) \in E_{1} \times E_{2}$. Take $(\phi, \psi)=\left(e^{(k)}, 0\right)$ and $(\phi, \psi)=$ $\left(0, e^{(k)}\right)$ in $(41)$ for $k \in \mathbb{Z}$, where

$$
e_{n}^{(k)}= \begin{cases}1, & n=k \\ 0, & n \neq k\end{cases}
$$

We see that $J^{\prime}\left(\phi^{*}, \psi^{*}\right)=0$. Thus, $\left(\phi^{*}, \psi^{*}\right)$ is a nontrivial solution to system (13). The proof is completed.

By Theorem 4, the system (1) has a nontrivial solution. However, it is uncertain if two components of this solution are nonzero. Therefore, we want to find solutions of the system (1) which have both of the components not identically zero. In order to achieve this goal, we consider the system (1) with $b_{1 n}=b_{2 n}, n \in \mathbb{Z}$; that is,

$$
\begin{aligned}
& i \frac{d u_{n}}{d t}=-(\mathscr{A} u)_{n}+b_{1 n} u_{n}-a_{1}\left|u_{n}\right|^{2} u_{n}-a_{3}\left|v_{n}\right|^{2} u_{n}, \\
& i \frac{d v_{n}}{d t}=-(\mathscr{A} v)_{n}+b_{1 n} v_{n}-a_{2}\left|v_{n}\right|^{2} v_{n}-a_{3}\left|u_{n}\right|^{2} v_{n} .
\end{aligned}
$$

In system (43), we know that $L_{1}=L_{2}$, where $L_{i}, i=1,2$, is given by (11). By the definition of $E_{i}, i=1,2$, in Section 2 of this paper, we obtain that $E_{1}=E_{2}$. Hence, $\lambda_{1}=\lambda_{2}$. For the sake of simplicity, we let $L_{1}=L_{2}=L, E_{1}=E_{2}=E$, and $\lambda_{1}=\lambda_{2}=\lambda$. The notations in Section 2, such as $J(\phi, \psi), I(\phi, \psi)$, and $N$ are the same.

Now, we give the second result of this paper as follows.

Theorem 5. Assume that $\omega_{1}<\lambda, \omega_{2}<\lambda, a_{3}>$ $\max \left\{a_{1}, a_{2},\left(\left(\lambda-\omega_{2}\right) /\left(\lambda-\omega_{1}\right)\right) a_{1},\left(\left(\lambda-\omega_{1}\right) /\left(\lambda-\omega_{2}\right)\right) a_{2}\right\}$, and (10) holds. Then system (43) has a nontrivial standing wave solution $(\widetilde{\phi}, \widetilde{\psi})$ in $E \times E$ with $\widetilde{\phi} \neq 0$ and $\widetilde{\psi} \neq 0$.

Proof. By Theorem 4, we know that system (43) has a nontrivial standing wave solution $(\widetilde{\phi}, \widetilde{\psi})$ in $E \times E$.

Now we will prove that $\widetilde{\phi} \neq 0$ and $\widetilde{\psi} \neq 0$.

Since $(\widetilde{\phi}, \widetilde{\psi}) \in N$, we know that $(\widetilde{\phi}, \widetilde{\psi}) \neq(0,0)$. If one of the components $(\widetilde{\phi}, \widetilde{\psi})$, say $\widetilde{\psi}=0$, then $\widetilde{\phi} \neq 0$. For $\epsilon$ small enough, we consider $(\widetilde{\phi}, \epsilon \widetilde{\phi}) \in(E-\{0\}) \times(E-\{0\})$; by a similar argument to the proof of Lemma 2, we know that there exists $t^{*}$ such that $I\left(t^{*} \widetilde{\phi}, t^{*} \epsilon \widetilde{\phi}\right)=0$; that is, $\left(t^{*} \widetilde{\phi}, t^{*} \in \widetilde{\phi}\right) \in N$.
By (20) and $I\left(t^{*} \widetilde{\phi}, t^{*} \in \widetilde{\phi}\right)=0$, we have $\left(t^{*}\right)^{2}=$ $\left(H_{1}(\widetilde{\phi}, \epsilon \widetilde{\phi})\right) /\left(H_{2}(\widetilde{\phi}, \epsilon \widetilde{\phi})\right)$, where

$$
\begin{gathered}
H_{1}(\phi, \psi)=\|\phi\|_{E}^{2}-\omega_{1}\|\phi\|_{l^{2}}^{2}+\|\psi\|_{E}^{2}-\omega_{2}\|\psi\|_{l^{2}}^{2}, \\
H_{2}(\phi, \psi)=\sum_{n \in \mathbb{Z}}\left(a_{1} \phi_{n}^{4}+a_{2} \psi_{n}^{4}+2 a_{3} \phi_{n}^{2} \psi_{n}^{2}\right),
\end{gathered}
$$

and $J\left(t^{*} \widetilde{\phi}, t^{*} \epsilon \widetilde{\phi}\right)=\left(H_{1}^{2}(\widetilde{\phi}, \epsilon \widetilde{\phi})\right) /\left(4 H_{2}(\widetilde{\phi}, \epsilon \widetilde{\phi})\right)$.

We noticed that $J(\widetilde{\phi}, 0)=\left(a_{1} / 4\right) \sum_{n \in \mathbb{Z}} \widetilde{\phi}^{4}=$ $\inf _{(\phi, \psi) \in N} J(\phi, \psi)$ and

$$
\begin{aligned}
H_{2}(\widetilde{\phi}, 0) & =H_{1}(\widetilde{\phi}, 0)=\|\widetilde{\phi}\|_{E}^{2}-\omega_{1}\|\widetilde{\phi}\|_{l^{2}}^{2} \\
& \geq\left(\lambda-\omega_{1}\right)\|\widetilde{\phi}\|_{l^{2}}^{2} .
\end{aligned}
$$

For the sake of simplicity, we let

$$
B=\sum_{n \in \mathbb{Z}} \widetilde{\phi}_{n}^{4}, \quad D=\|\widetilde{\phi}\|_{E}^{2}-\omega_{2}\|\widetilde{\phi}\|_{l^{2}}^{2} .
$$

If $\omega_{1} \leq \omega_{2}<\lambda$, then

$$
\begin{aligned}
D & =\|\widetilde{\phi}\|_{E}^{2}-\omega_{2}\|\widetilde{\phi}\|_{l^{2}}^{2} \\
& =\|\widetilde{\phi}\|_{E}^{2}-\omega_{1}\|\widetilde{\phi}\|_{l^{2}}^{2}+\left(\omega_{1}-\omega_{2}\right)\|\widetilde{\phi}\|_{l^{2}}^{2} \\
& =a_{1} B+\left(\omega_{1}-\omega_{2}\right)\|\widetilde{\phi}\|_{l^{2}}^{2} \leq a_{1} B .
\end{aligned}
$$

Thus, $a_{3}>a_{1}$ and (47) yields $a_{1} B D<a_{1} a_{3} B^{2}$.

If $\omega_{2}<\omega_{1}<\lambda$, then by (45),

$$
\begin{aligned}
D & =\|\widetilde{\phi}\|_{E}^{2}-\omega_{2}\|\widetilde{\phi}\|_{l^{2}}^{2} \\
& =\|\widetilde{\phi}\|_{E}^{2}-\omega_{1}\|\widetilde{\phi}\|_{l^{2}}^{2}+\left(\omega_{1}-\omega_{2}\right)\|\widetilde{\phi}\|_{l^{2}}^{2} \\
& =a_{1} B+\left(\omega_{1}-\omega_{2}\right)\|\widetilde{\phi}\|_{l^{2}}^{2} \\
& \leq a_{1} B+\frac{\omega_{1}-\omega_{2}}{\lambda-\omega_{1}} a_{1} B=\frac{\lambda-\omega_{2}}{\lambda-\omega_{1}} a_{1} B .
\end{aligned}
$$

Thus, $a_{3}>\left(\left(\lambda-\omega_{2}\right) /\left(\lambda-\omega_{1}\right)\right) a_{1}$ and (48) yields $a_{1} B D<$ $a_{1} a_{3} B^{2}$.

From the above arguments, if $a_{3}>\max \left\{a_{1},\left(\left(\lambda-\omega_{2}\right) /(\lambda-\right.\right.$ $\left.\left.\left.\omega_{1}\right)\right) a_{1}\right\}$, then $a_{1} B D<a_{1} a_{3} B^{2}$.

For $\epsilon$ small enough, we have

$$
\begin{aligned}
H_{1}^{2}(\widetilde{\phi}, \epsilon \widetilde{\phi}) \\
=\left(\|\widetilde{\phi}\|_{E}^{2}-\omega_{1}\|\widetilde{\phi}\|_{l^{2}}^{2}+\|\epsilon \widetilde{\phi}\|_{E}^{2}-\omega_{2}\|\epsilon \widetilde{\phi}\|_{l^{2}}^{2}\right)^{2} \\
=\left(a_{1} B+\epsilon^{2} D\right)^{2}=a_{1}^{2} B^{2}+2 a_{1} B D \epsilon^{2}+D^{2} \epsilon^{4} \\
\quad<a_{1}^{2} B^{2}+2 a_{1} a_{3} B^{2} \epsilon^{2}+a_{1} a_{2} B^{2} \epsilon^{4} \\
=a_{1} B\left(a_{1} B+a_{2} B \epsilon^{4}+2 a_{3} B \epsilon^{2}\right) \\
=H_{2}(\widetilde{\phi}, 0) H_{2}(\widetilde{\phi}, \epsilon \widetilde{\phi}) .
\end{aligned}
$$


Hence, by (49), we have

$$
\begin{aligned}
J\left(t^{*} \widetilde{\phi}, t^{*} \epsilon \widetilde{\phi}\right) & =\frac{H_{1}^{2}(\widetilde{\phi}, \epsilon \widetilde{\phi})}{4 H_{2}(\widetilde{\phi}, \epsilon \widetilde{\phi})}<\frac{1}{4} H_{2}(\widetilde{\phi}, 0) \\
& =J(\widetilde{\phi}, 0)=\inf _{(\phi, \psi) \in N} J(\phi, \psi) .
\end{aligned}
$$

This is a contradiction. So, $\widetilde{\psi} \neq 0$.

Similarly, if $\widetilde{\psi} \neq 0$ and $a_{3}>\max \left\{a_{2},\left(\left(\lambda-\omega_{1}\right) /\left(\lambda-\omega_{2}\right)\right) a_{2}\right\}$, then $\widetilde{\phi} \neq 0$. The proof is completed.

\section{Acknowledgments}

This work is supported by Program for Changjiang Scholars and Innovative Research Team in University (no. IRT1226), the National Natural Science Foundation of China (no. 11171078), and the Specialized Fund for the Doctoral Program of Higher Education of China (no. 20114410110002).

\section{References}

[1] J. Belmonte-Beitia, V. M. Pérez-García, and P. J. Torres, "Solitary waves for linearly coupled nonlinear Schrödinger equations with inhomogeneous coefficients," Journal of Nonlinear Science, vol. 19, no. 4, pp. 437-451, 2009.

[2] C. J. Myatt, E. A. Burt, R. W. Ghrist, E. A. Cornell, and C. E. Wieman, "Production of two overlapping Bose-Einstein condensates by sympathetic cooling," Physical Review Letters, vol. 78, no. 4, pp. 586-589, 1997.

[3] R. S. MacKay and S. Aubry, "Proof of existence of breathers for time-reversible or Hamiltonian networks of weakly coupled oscillators," Nonlinearity, vol. 7, no. 6, pp. 1623-1643, 1994.

[4] S. Aubry, "Breathers in nonlinear lattices: existence, linear stability and quantization," Physica D, vol. 103, no. 1-4, pp. 201250, 1997, Lattice dynamics (Paris, 1995).

[5] P. G. Kevrekidis, The Discrete Nonlinear Schrödinger Equation, Springer, 2009

[6] D. E. Pelinovsky and V. M. Rothos, "Bifurcations of travelling wave solutions in the discrete NLS equations," Physica D, vol. 202, no. 1-2, pp. 16-36, 2005.

[7] A. Pankov and V. Rothos, "Periodic and decaying solutions in discrete nonlinear Schrödinger with saturable nonlinearity," Proceedings of The Royal Society of London A, vol. 464, no. 2100, pp. 3219-3236, 2008.

[8] H. Shi and H. Zhang, "Existence of gap solitons in a periodic discrete nonlinear Schrödinger equations," Journal of Mathematical Analysis and Applications, vol. 371, no. 1, pp. 254-265, 2010.

[9] G. Zhang and F. Liu, "Existence of breather solutions of the DNLS equations with unbounded potentials," Nonlinear Analysis. Theory, Methods \& Applications A, vol. 71, no. 12, pp. e786-e792, 2009.

[10] Z. Zhou and J. Yu, "On the existence of homoclinic solutions of a class of discrete nonlinear periodic systems," Journal of Differential Equations, vol. 249, no. 5, pp. 1199-1212, 2010.

[11] Z. Zhou, J. Yu, and Y. Chen, "On the existence of gap solitons in a periodic discrete nonlinear Schrödinger equation with saturable nonlinearity," Nonlinearity, vol. 23, no. 7, pp. 17271740, 2010.
[12] L. Liu, W. Yan, and X. Zhao, "The existence of standing wave for the discrete coupled nonlinear Schrödinger lattice," Physics Letters A, vol. 374, no. 15-16, pp. 1690-1693, 2010. 


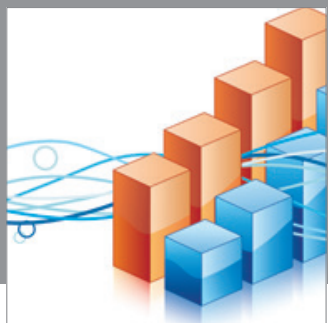

Advances in

Operations Research

mansans

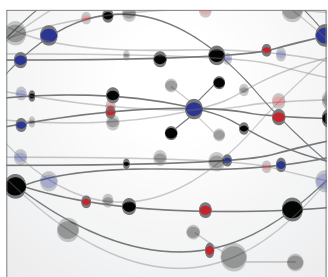

The Scientific World Journal
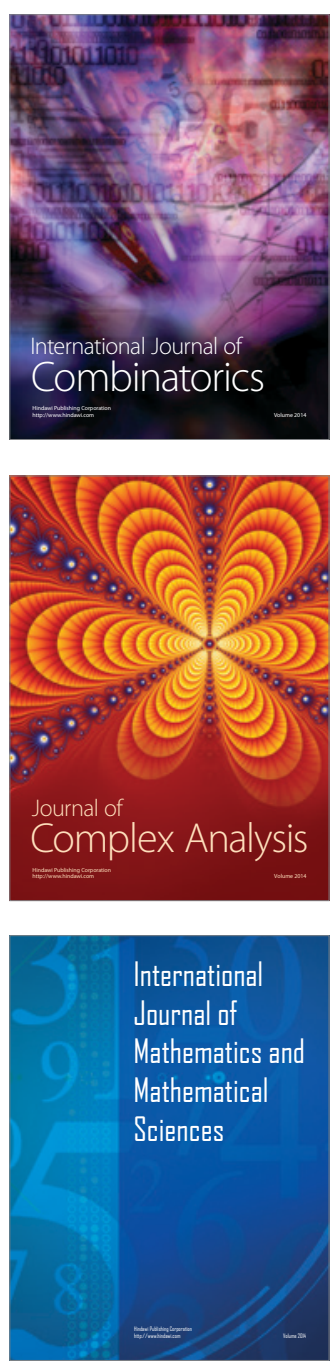
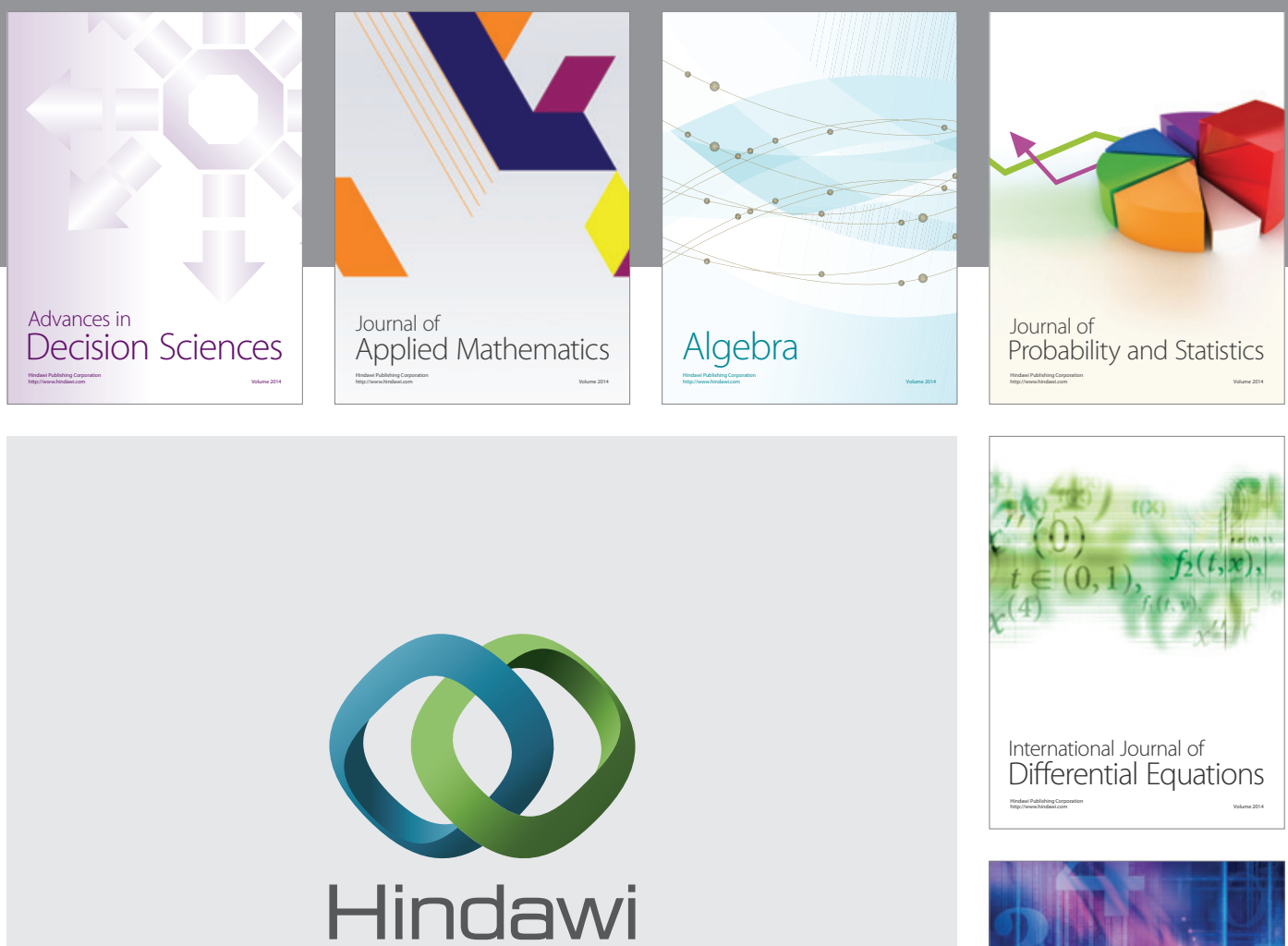

Submit your manuscripts at http://www.hindawi.com
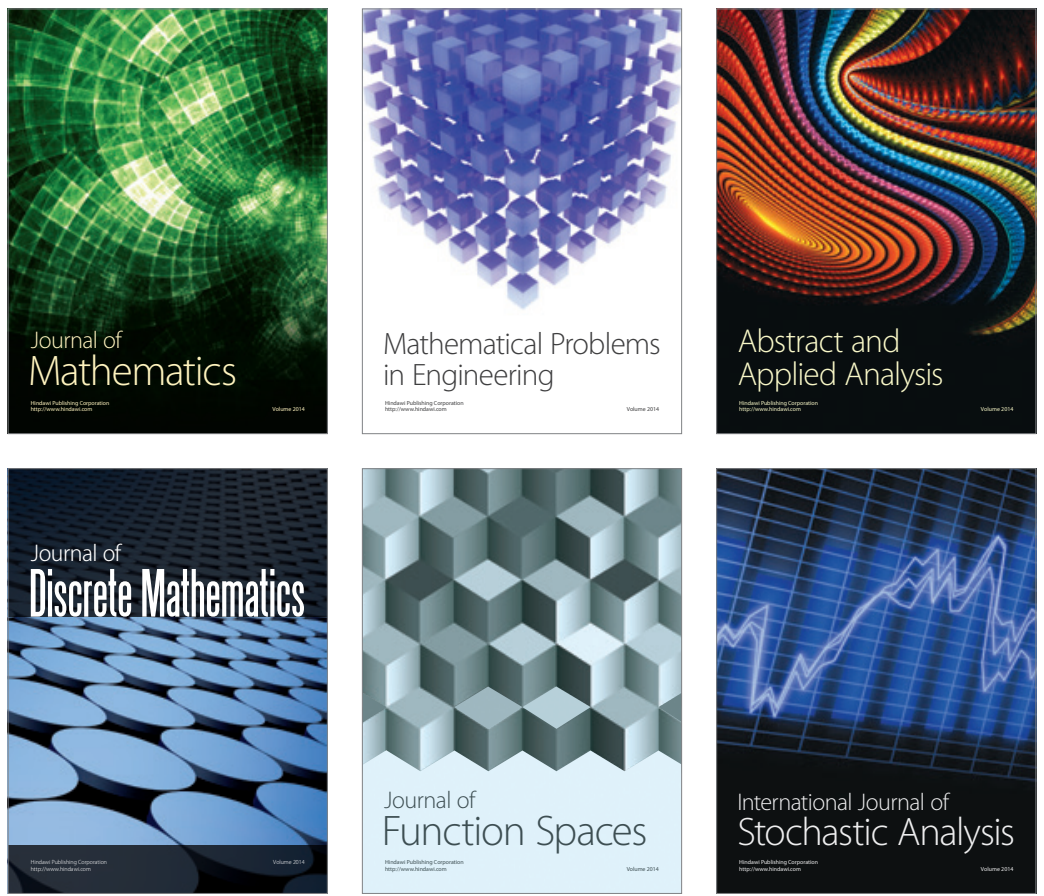

Journal of

Function Spaces

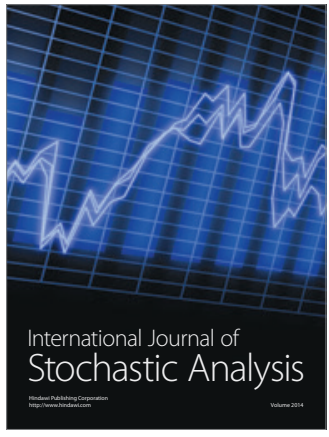

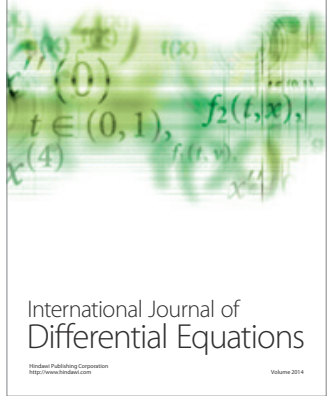
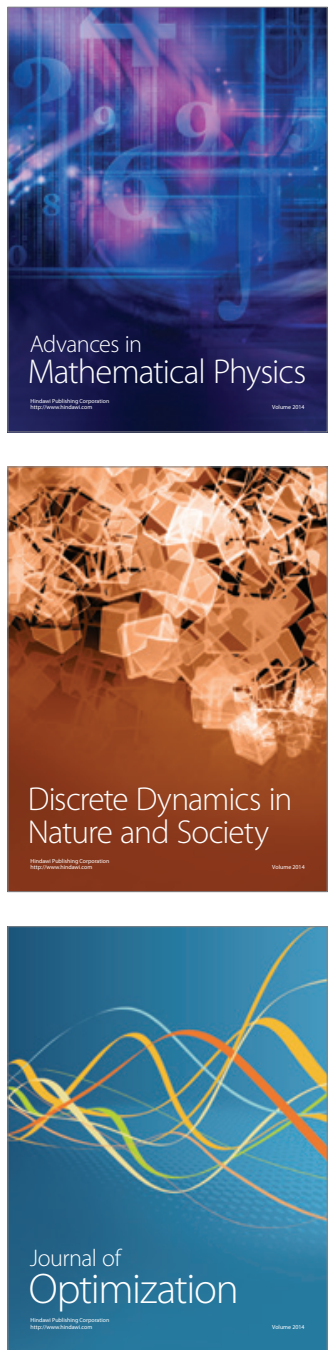